\title{
城市化对杭州市鸟类营巢集团的影响
}

\author{
李 鹏, 张竞成, 李必成, Chris Wood, 蒋萍萍, 丁 平 ${ }^{*}$ \\ (浙江大学 生命科学学院, 浙江 杭州 310058)
}

摘要: 为了了解城市化对鸟类营巢集团的影响, 2007 年 3 月至 2007 年 8 月, 沿城市化梯度在杭州地区的城 区、城乡结合区、农田区、农林交错区和自然林区等类型栖息地内设置 $3000 \mathrm{~m}$ 长、 $100 \mathrm{~m}$ 宽的样线各 18 条，共 计 90 条; 并对其鸟类物种与群落营巢集团进行调查。调查共记录到繁殖鸟类 96 种, 根据鸟类营巢位置可将其分 为林冠枝干营巢集团、灌草丛营巢集团、林冠枝干/灌草丛营巢集团、自然洞穴营巢集团、人工建筑营巢集团、自 然洞穴/人工建筑营巢集团地面营巢集团、水面营巢集团和寄生集团共 9 种。进一步分析显示, 随城市化程度提高, 鸟类营巢集团的数目呈整体下降趋势，而各集团的物种数也基本呈下降趋势。但是，不同的营巢集团对城市化的 反应存在差异, 导致其物种组成在群落中比例的明显变化。城市化程度的提高使树冠筑巢鸟、灌从筑巢鸟、地面 筑巢鸟及自然洞穴筑巢鸟在群落中所占的比例明显下降, 其中地面筑巢鸟最为敏感, 灌从筑巢鸟次之。同时, 自 然洞穴/人工建筑集团的鸟类在群落中的比例却随着城市化程度的提高明显上升。各营巢集团之所以对城市化做出 不同反应, 是因为它们对筑巢地资源利用方式不同。此外, 还发现植被盖度、人工设施面积、至市中心距离以及 人为干扰等因素均可对鸟类营巢集团结构产生影响, 但不同类型的营巢集团对上述因素的反应各异。

关键词: 城市化; 鸟类; 营巢集团; 杭州

中图分类号：Q959.7 文献标识码：A 文章编号：0254-5853-(2009)03-0295-08

\section{Effects of Urbanization on the Avian Nesting Guilds in Hangzhou}

\author{
LI Peng, ZHANG Jing-cheng, LI Bi-cheng, Chris Wood, JIANG Ping-ping, DING Ping* \\ (College of Life Sciences, Zhejiang University, Hangzhou 310058, China)
}

\begin{abstract}
We investigated the avian nesting guilds in Hangzhou across an urban gradient from March 2007 to August 2007. This gradient includes different urbanization level habitats of urban areas, rural-urban continuum areas, farmland areas, forestland mixed areas, and forestland areas, in order to recognize the effects of urbanization on avian nesting guilds. There were 18 strip transects $(3 \mathrm{~km} \times 100 \mathrm{~m})$ in each type of habitats, totaling 90 transects. Ninety-six bird species and 9 nesting guilds were recorded. Based on the locations of the nest sites, these were classified as canopy nesters, shrubs nesters, canopy/ shrub nesters, natural cavity nesters, buildings nesters, natural cavity/ buildings nesters, ground nesters, water surface nesters and parasite nesters. The results showed that the urbanization could decrease both the numbers of the nesting guilds and the species in nesting guilds. We found that the urbanization had different impact patterns for different nesting guilds. For example, the number of canopy nesters, shrub nesters, ground nesters and natural cavity nesters declined with increased urbanization. Shrub nesters and ground nesters were particularly more sensitive to urbanization. For the natural cavity/build nesters, numbers were increased with urbanization. The affecting patterns of urbanization were different with different nesting guilds as they using different nest-sites. Additionally, we found that although all of variables of habitat measured in our survey, the vegetation coverage, areas of buildings, distance to the city center and human disturbance could influence the avian community structure of nesting guilds, each variable had a specific pattern of the effect on the nesting guilds separately.
\end{abstract}

Key words: Urbanization; Birds; Nesting guilds; Hangzhou

城市化是指人类向住宅区和工业区不断集中 的过程 (Chace \& Walsh, 2006)。它不仅是土地利用

收稿日期: 2009-02-27; 接受日期: 2009-05-04

基金项目：国家自然科学基金（30670344); 杭州市林业水利局资助项目

“通讯作者 (Corresponding author), E-mail: dingping@zju.edu.cn

第一作者简介: 李鹏, 男, 硕士研究生, 主要从事鸟类生态学研究 
方式的改变, 而且将会导致动物栖息地发生深刻的 改变, 进而对野生动物种群和群落产生影响。随着 全球人口快速增长、世界经济的迅速发展和城市化 进程的加快, 城市化对野生动物影响的问题, 尤其 是对鸟类的影响已引起人们的高度关注（Blair, 1996; Rottenborn, 1999; Mckinney, 2002)。因此, 有 大量的研究涉及到城市化对鸟类影响的问题 (Chace \& Walsh, 2006)。这些研究发现, 随着城市 化程度的提高, 鸟类群落的物种丰富度和多样性会 随之降低 (Beissinger \& Osborne, 1982; Zhuge \& Jiang, 1983; DeGraaf \& Wentworth, 1986; Rosenberg et al, 1987; Rottenborn, 1999; Melles et al, 2003)。由 于城市化会增加了人工植被和人工食物等额外资 源 (Blair, 1996), 因此又有研究认为适度的人类干 扰对鸟类群落的多样性和丰富度无有害影响

(Palomino \& Carrascal, 2007), 甚至有学者认为适 度的城市化反而有助于提高鸟类群落的物种丰富 度和多样性（Mills et al，1989; Blair，1996，1999， 2004 ), 鸟类密度亦可能与城市化程度呈正相关关 系 (Clergeau et al, 1998; Palomino \& Carrascal, 2006)。

城市化不仅影响鸟类的物种多样性和丰富度, 也影响着鸟类群落的结构。从集团的角度来看, 鸟 类取食集团中的食虫鸟类集团物种数往往随着城 市化程度的提高而减少 (Clergeau et al, 1998; Allen \& O'Connor, 2000; Lindsay et al, 2002; Wang et al, 2004 ); 而杂食性鸟类集团的物种数却与城市化程 度呈正相关关系 (Jokimäki \& Suhonen, 1998)。在 营巢集团方面, 地面营巢集团和灌从营巢集团受城 市化影响较大, 其物种数在城市化程度高的地区明 显减少（Luniak，1981; Jokimäki，1999; Fern á ndez-Juricic, 2000); 树冠营巢集团受到的影响次之, 而洞穴营巢集团对城市化最不敏感（Park \& Lee, 2000)。

然而, 虽然过去已有不少涉及城市化对鸟类营 巢集团影响及其影响模式的研究, 但大多数研究都 在温带及热带地区进行。亚热带季风气候区是全球 人口密度最高及人口增长速度最快的区域之一, 而 有关该区域内城市化对鸟类影响的研究却相对不 足 (Chace \&Walsh, 2006), 其中人们对于城市化对 鸟类营巢集团的影响模式更是知之甚少。而在相对 较高的人类干扰水平及城市化压力下, 城市化对鸟 类营巢集团或许存在不同的影响模式。为此, 本研
究拟通过对整个杭州地区不同城市化梯度的栖息 地与鸟类营巢集团关系的研究, 验证: (1) 随着城 市化程度的提高，鸟类集团数量将减少;（2）城市 化对鸟类地面营巢集团的影响最大, 灌从营巢集团 次之, 树冠营巢集团再次之, 而对洞穴营巢集团影 响最低; (3) 植被盖度与鸟类营巢集团的变化密切 相关 (Green \& Baker, 2003) 等假设, 进而探讨亚 热带季风气候地区城市化对鸟类营巢集团的影响 模式, 为这区域内城市鸟类多样性的保护提供科学 依据。

\section{1 研究方法}

\section{1 研究地点}

本研究区域为浙江省杭州市 (包括杭州市区、 富阳市、桐庐县、临安市、建德市和淳安县), 位 于中国东南部沿海地区, 面积 $16596 \mathrm{~km}^{2}$, 地理坐 标为北纬 $29^{\circ} 12^{\prime}-30^{\circ} 35^{\prime}$, 东经 $118^{\circ} 12^{\prime}-120^{\circ}$ 43 '。杭州市西北部和西南部系浙西中山丘陵区, 东 北部和东南部则属浙北平原。全市丘陵山地占总面 积的 $65.6 \%$, 平原占 $26.4 \%$, 江、河、湖、荡、水 库占 $8 \%$ 。最高峰为临安市西部与安徽省交界的清 凉峰, 海拔 $1787 \mathrm{~m}$ 。杭州市处于中亚热带常绿阔叶 林植被带, 平均森林覆盖率为 $62.8 \%$ 。

\section{2 调查样区}

本研究按自然环境梯度, 将研究区域划分为城 区、城乡结合区、农田区、农林交错区、以及自然 林区 5 类样区。

城区位于杭州市各县市中心城市市区, 其区域 内建筑物高大且集中, 人口密集, 交通繁忙, 人为 活动干扰严重。植被多为人工种植, 总体上面积小 并呈斑块状分布, 并且在垂直结构上缺乏灌木层和 草本层。不过城区的景观异质性较高, 边界也较多。

城乡结合区位于中心城市外围, 其建筑物不如 城区高大，密度也相对较低。一般大型工厂，厂房 多位于该区域, 人为活动干扰强烈, 人口相对密集。 植被面积小, 且多为农田或杂草荒地。

农田区位于各县市地势较为平坦的农村地区, 建筑物少且低矮, 多为农民住房, 虽村落较多, 但 总体人为活动干扰不大。植被构成多为农田, 也有 少量果树林, 一些区域还分布有零星的次生林。

农林交错区相对与农区更加远离中心城市，一 般位于山区, 地势较高且不平坦, 交通不便。建筑 物仅为农民住房, 村落密度小于农区, 人为活动干 
扰较小。植被构成除了农田, 果树林外, 还有大量 的次生针阔混交林。

自然林区离中心城市最远, 地势最高, 人工建 筑很少, 人为活动干扰最小。植被覆盖率高, 大部 分为地区次生常绿落叶阔叶混交林, 某些地区还保 留有完好的原始自然植被, 也有少量的竹林和小块 的农田。

本研究在城区、城乡结合区、农田区、农林交 错区及自然林区等样区各选取样带 18 条, 总计 90 条。每条样带长 $3 \mathrm{~km}$, 带宽每边 $50 \mathrm{~m}$, 各样带之间 间隔大于 $4 \mathrm{~km}$ (城区内各样带之间间隔大于 $1 \mathrm{~km}$ )。

\section{3 乌类调查}

在 2007 年 3 月至 2007 年 8 月期间, 对选定的 90 条样线进行调查, 每条样线调查一次。调查选在 天气晴朗、少雾、无大风的日子进行, 调查时间一 般为上午 6:00-10:00 时和下午 16:00-18:00 时。 调查时由 $2-3$ 人组成一个小组, 步行调查, 使用 8 -10 倍双目望远镜对鸟类进行观察。记录样线两侧 见到或听到的鸟类种类与数量, 并记录鸟类的求偶 炫耀行为、领域行为、筑巢行为、孵卵行为及育雏 行为等。

\section{4 城市化特征参数选取和测量}

在以往的城市鸟类生态学的研究中, 研究者使 用过多种城市化特征参数, 如植被盖度 (Rottenborn, 1999)、至城市边界距离 (Bolger et al, 1997; Wang et al, 2008)、建筑比例（Mills et al, 1989; Blair, 1996; Bolger et al, 1997; Lim \& Sodhi, 2004; Palomino \& Carrascal, 2006)、道路比例 (Palomino \& Carrascal, 2006)、人行道数量 (Mills et al, 1989; Blair, 1996)、 环境噪音 (Wang et al, 2008) 和人口密度 (Lim \& Sodhi, 2004) 等, 这些参数都从不同侧面反映了栖 息地的城市化特征。但是在城市化的过程中，栖息 地的景观改变非常复杂, 单一的栖息地城市化特征 参数往往很难真正反映栖息地的城市化程度, 因此 本研究选取至中心城市市中心距离、人为干扰、植 被盖度和人工设施指数等 4 项城市化特征参数, 并 在此基础上参考 Chen et al（2000）的方法, 综合以 上参数提出城市化综合指数。各项参数的测量及说 明见表 1 。

\section{5 营巢集团分类}

鸟类营巢类型的调查方法采用实地调查与查 找文献相结合的方法, 即在野外调查中, 若发现有

表 1 杭州地区城市化特征参数测量及说明

Tab. 1 Measurements and introductions of urbanization characteristic parameters in Hangzhou

\begin{tabular}{|c|c|}
\hline 至中心城市市中心距离 Distance to city center & $\begin{array}{l}\text { 选定杭州市主城、临平市、萧山市、富阳市、桐庐县城、临安市、建德市和千 } \\
\text { 岛湖镇等各县市行政机构所在地为中心城市, 根据地图估测样带至最近中心城 } \\
\text { 市市中心的距离, 单位: } \mathrm{km}\end{array}$ \\
\hline 人为干扰 Human disturbance & $\begin{array}{l}\text { 表示人为干扰的程度, 分为 } 6 \text { 个等级 }(0-5): 0 \text { 表示样带内无人; } 1 \text { 表示样带 } \\
\text { 内有 } 1-2 \text { 人; } 2 \text { 表示样带内有 } 3-5 \text { 人; } 3 \text { 表示条样带内有 } 6-10 \text { 人; } 4 \text { 表示样 } \\
\text { 带内有 } 10-20 \text { 人; } 5 \text { 表示样带内有 } 20 \text { 人以上, 其中对生境类型复杂的样带进行 } \\
\text { 分段赋值然后取其加权平均数 }\end{array}$ \\
\hline 植被盖度 Vegetation coverage & 表示植被在样带内所占面积的比率，野外调查时估测，以百分比表示 \\
\hline 人工设施指数 Building index & 表示人工设施在样带内所占面积的比率，野外调查时估测，以百分比表示 \\
\hline 城市化综合指数 Urbanization synthetical index & $\begin{array}{l}\text { 表示城市化的程度, 为了综合反映以上 } 4 \text { 项城市化特征参数, 计算中将它们调 } \\
\text { 整为随城市化程度的提高而在 } 0-100 \text { 之间递增的数值。其中样带距中心城市最 } \\
\text { 远的距离为 } 46.5 \mathrm{~km} \text {, 将其定义为 } 0 \text {, 故距中心城市距离指数定义为 }\{100- \\
(100 / 46.5) \times \text { 距中心城市距离 } \mathrm{km}\} \text {, 即 }(100-2.15 \times \text { 距中心城市距离 } \mathrm{km}) \text {; 人为 } \\
\text { 干扰各等级乘以 } 20 \text {; 植被盖度乘以 } 100 \text {, 并用 } 100 \text { 减之; 人工设施指数乘以 } 100 \text { 。 } \\
\text { 于是城市化综合指数 }=(100-2.15 \times \text { 距中心城市距离 } \mathrm{km})+(20 \times \text { 干扰程度 })+(100 \text { (设施指数 }) \text { 。 } \\
-100 \times \text { 植被盖度 })+(100 \times \text { 人 }\end{array}$ \\
\hline
\end{tabular}

鸟类在巢中栖息、繁殖、孵卵或育倠, 即记录此巢 的位置与类型，对于未实地观察到鸟巢的鸟类，其 营巢类型则根据文献 (Cheng et al, 1978, 1979, 1987, 1991, 1995, 1997; Li et al, 1982, 2004; Zheng, 1985; Zheng, 1995; Chen \& Luo, 1998; Fu et al, 1998; Sun et al, 2002; Li, 2004) 确认。

根据已有的鸟类集团分类方法 (Canterbury et al, 2000; Fernández-Juricic, 2000; Park \& Lee, 2000; Lindsay et al, 2002; Lim \& Sodhi, 2004; Palomino \& Carrascal, 2007; Fontaine et al, 2007) 和本研究实际 情况, 本文将调查记录到的繁殖鸟类按照营巢位置 分为林冠枝干营巢集团 (canopy nesters)、灌草从营 巢集团 (shrubs nesters)、林冠枝干/灌草丛营巢集团 ( canopy/ shrub nesters)、自然洞穴营巢集团 
( natural cavity nesters)、人工建筑营巢集团 (buildings nesters)、自然洞穴/人工建筑营巢集团 (natural cavity/ buildings nesters)、地面营巢集团 (ground nesters)、水面营巢集团（water surface nesters) 和寄生集团 (parasite nesters) 等 9 种群团。 每种鸟类只归属于一种营巢集团。

\section{6 数据分析}

计算出各栖息地中鸟类营巢集团数的平均数、 各营巢集团的总物种数以及各营巢集团的平均比 率, 然后对鸟类营巢集团数的平均数和各营巢集团 的平均比率进行单因子方差分析 (one-way ANOVA), 以确定鸟类营巢集团数的平均数及各营 巢集团的平均比率在不同栖息地中是否存在显著 差异。此外, 通过鸟类营巢集团平均比率的与不同 栖息地的城市化特征参数的直线回归分析 (linear regression analysis), 来探讨影响鸟类营巢集团分布
的主要因子。以上分析均采用 SPSS 13.0 for Windows 软件进行。

\section{2 结 果}

\section{1 不同栖息地的城市化梯度分析}

在对城区、城乡结合区、农田区、农林交错区 和自然林区等样区的各项城市化特征参数进行测 量计算后，发现沿城区、城乡结合区、农田区、农 林交错区和自然林区的梯度, 植被盖度和至中心城 市市中心距离两项参数的平均值递增, 而人为干扰 和人工设施指数的平均值则递减（表 2)。

通过对各样区的城市化综合指数的计算可知, 城区的城市化程度最高 $(382.00 \pm 6.91)$, 随后依次 为城乡集合区 $(305.51 \pm 6.97)$ ，农田区（248.49士 $4.85)$ ，农林交错区（174.35 $\pm 8.10 ）$, 最低的是自然

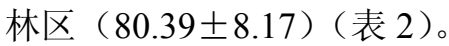

表 2 杭州地区不同栖息地城市化特征参数的平均值

Tab. 2 Mean values $(M \pm S E)$ of urban characteristic parameters of different habitats in Hangzhou

\begin{tabular}{|c|c|c|c|c|c|}
\hline $\begin{array}{c}\text { 城市化特征参数 } \\
\text { Urban characteristic parameters }\end{array}$ & $\begin{array}{c}\text { 城区 } \\
\text { Urban areas }\end{array}$ & $\begin{array}{c}\text { 城乡结合区 } \\
\text { Rural-urban continuum } \\
\text { areas }\end{array}$ & $\begin{array}{l}\text { 农田区 } \\
\text { Farmland } \\
\text { areas }\end{array}$ & $\begin{array}{c}\text { 农林交错区 } \\
\text { Farmland and forestland mixed } \\
\text { areas }\end{array}$ & $\begin{array}{c}\text { 自然林区 } \\
\text { Forestland } \\
\text { areas }\end{array}$ \\
\hline $\begin{array}{l}\text { 至中心城市市中心距离（km） } \\
\text { Distance to city center }\end{array}$ & $2.10 \pm 0.29$ & $10.42 \pm 1.04$ & $12.56 \pm 10.8$ & $19.38 \pm 1.89$ & $28.01 \pm 2.78$ \\
\hline $\begin{array}{l}\text { 人为干扰 } \\
\text { Human disturbance }\end{array}$ & $4.93 \pm 0.08$ & $3.76 \pm 0.18$ & $2.39 \pm 0.19$ & $1.31 \pm 0.21$ & $0.46 \pm 0.09$ \\
\hline $\begin{array}{l}\text { 植被盖度（\%) } \\
\text { Vegetation coverage }\end{array}$ & $11.61 \pm 1.88$ & $39.67 \pm 3.37$ & $62.94 \pm 3.14$ & $71.83 \pm 3.78$ & $84.44 \pm 2.28$ \\
\hline $\begin{array}{l}\text { 人工设施指数（\%) } \\
\text { Building index }\end{array}$ & $94.06 \pm 1.12$ & $91.28 \pm 1.69$ & $90.72 \pm 1.40$ & $62.72 \pm 2.63$ & $17.72 \pm 3.52$ \\
\hline $\begin{array}{l}\text { 城市化综合指数 } \\
\text { Urbanization synthetic index }\end{array}$ & $382.00 \pm 6.91$ & $305.51 \pm 6.97$ & $248.49 \pm 4.85$ & $174.35 \pm 8.10$ & $80.39 \pm 8.17$ \\
\hline
\end{tabular}

\section{2 不同栖息地鸟类营巢集团的分布及组成}

本研究在 5 类栖息地中共发现鸟类 96 种, 分 别属于 9 个营巢集团。其中城区平均有 $4.06 \pm 1.06$ 个营巢集团, 城乡结合区平均有 $4.28 \pm 1.02$ 个营巢 集团, 农田区平均有 $5.56 \pm 0.86$ 个营巢集团, 农林 交错区平均有 $6.22 \pm 1.00$ 个营巢集团, 以及自然林 区平均有 $6.89 \pm 1.02$ 个营巢集团 $(P<0.001$, one-way ANOVA)。可见, 随城市化程度的提高, 鸟类营巢 集团的数目呈下降趋势（图 1)。

随着城市化程度的提高, 林冠枝干营巢集团和 灌草丛营巢集团的鸟类物种数呈下降趋势, 其中灌 草丛营巢集团的趋势更为明显; 而林冠枝干/灌草丛 营巢集团物种数基本不变, 只是在城区略高; 自然 洞穴营巢集团的鸟类物种数也随之降低, 特别是从
自然林区到农林交错区，其降低趋势明显; 人工建 筑营巢集团的鸟类物种数基本不变; 自然洞穴/人工 建筑营巢集团的物种数变化也不大; 地面营巢集团 的鸟类数量较少，在城区没有分布，在农田区和城 乡结合区分布较少，在自然林区与农林交错区则略 多一些; 水面营巢集团的鸟类更少，只在农林交错 区和自然林区各分布有一种，即小丣挀；寄生集团 主要是鹃形目鸟类，在城区和城乡结合区无分布， 在剩余 3 种栖息地中物种数基本相同, 其中农林交 错区和自然林区略多 (图 2)。

在不同栖息地内, 鸟类营巢集团的组成存在显 著差异 $(P<0.05$, one-way ANOVA)。在城区和城 乡结合区, 利用人工建筑筑巢的鸟类集团, 即人工 建筑营巢集团与自然洞穴/人工建筑营巢集团的鸟 


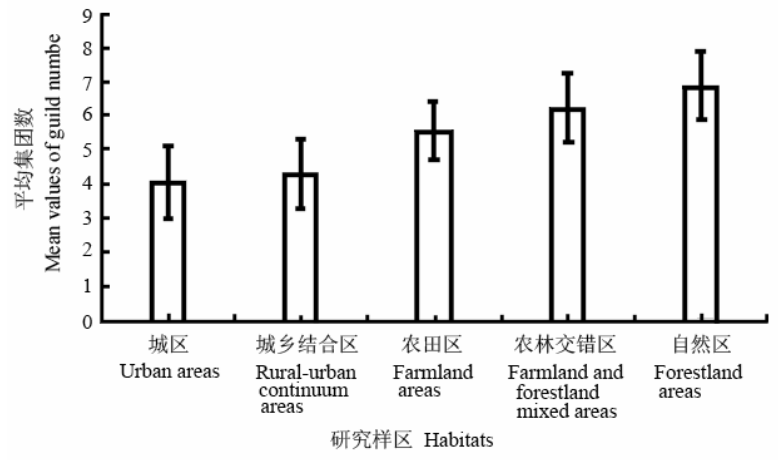

图 1 各栖息地平均集团数

Fig. 1 Mean values $(\mathrm{M} \pm S D)$ of guild number in different habitats

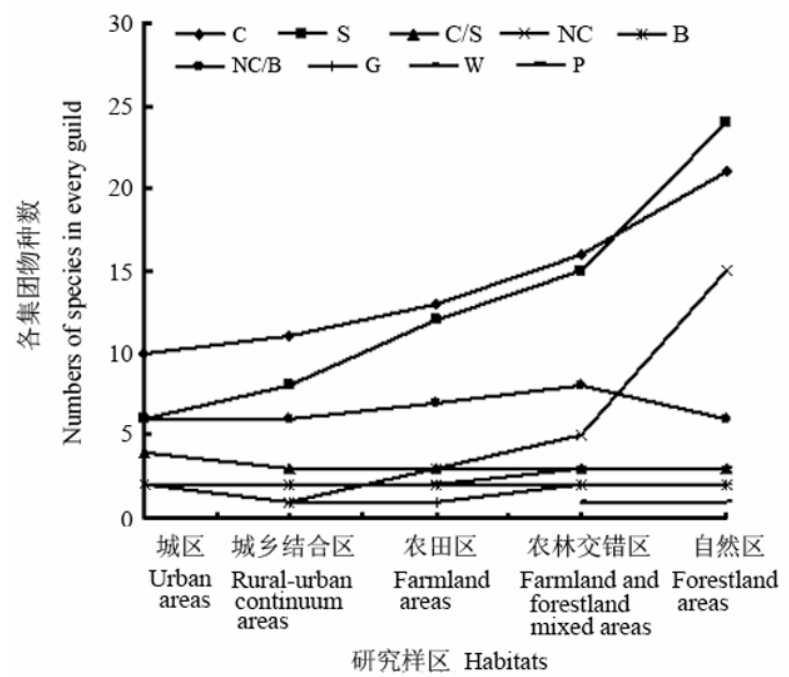

图 2 不同栖息地的各集团物种数

Fig. 2 Numbers of species in every guild in different habitats C：林冠枝干营巢集团 (canopy nesters); S: 灌草丛营巢集团 (shrubs nesters); C/S: 林冠枝干/灌草丛营巢集团 (canopy/ shrub nesters); NC： 自然洞穴营巢集团 (natural cavity nesters); B：人工建筑营巢集团 (buildings nesters); NC/B: 自然洞穴/人工建筑营巢集团 (natural cavity/ buildings nesters); G: 地面营巢集团 (ground nesters); W: 水面营巢 集团（water surface nesters）; P: 寄生集团（parasite nesters）。

类数量占多数; 在农田区, 自然洞穴/人工建筑营巢 集团的鸟类数量也占多数，但相对于前两个区域鸟 类数量所占的比率有所下降; 在农林交错区与自然 林区，则是灌草丛营巢集团所占的比率最大，不同 的是在农林交错区排第二位的是自然洞穴/人工建 筑营巢集团, 而在自然林区则是林冠枝干营巢集团 (表 3)。

随着城市化程度降低, 林冠枝干营巢集团、灌 草丛营巢集团和地面营巢集团所占的比例稳步上 升 $(P<0.05$, one-way ANOVA), 而自然洞穴营巢 集团与自然洞穴/人工建筑营巢集团的比例则呈下
降趋势 $(P<0.05$, one-way ANOVA)。林冠枝干/灌 草丛营巢集团在城区所占比例较高，在其余 4 类栖 息地比例有所下降且变化不大 $(P=0.07$, one-way ANOVA）。而人工建筑营巢集团则在农田区达到所 占比例的最大值, 但其趋势并不显著 (表 3)。

\section{3 影响鸟类营巢集团分布的主要因子}

6 种营巢集团在各栖息地中的平均比率与栖息 地参数的直线回归分析（地面营巢集团、水面营巢 集团及寄生集团因数据太少未进行此项分析）显示 （表 4)，林冠枝干营巢集团的平均比率与各项城市 化特征参数之间都存在显著的直线回归关系（表 4); 而灌草丛营巢集团及自然洞穴/人工建筑营巢集 团则与除植被盖度以外的各项城市化特征参数存 在显著直线回归相关（表 4)。

从表 4 可以看出人工设施所占面积的比例对鸟 类营巢集团分布的影响最为明显, 而至市中心距离 和人为干扰程度对各营巢集团的分布也有很大的 影响。此外植被盖度对依赖植物筑巢的营巢集团也 有明显的影响。

\section{3 讨 论}

\section{1 乌类营巢集团对城市化的反应}

栖息地在城市化后，植被面积大大减少，而植 被与鸟类的多样性和丰富度密切相关 (Goldstein, 1986; Mills et al, 1989; Green \& Baker, 2003; MacGregor-Fors, 2007)。并且，城市中的植被结构 和树种都较为单一, 多样性差, 虽然城市中也有一 些较好的林地，但总体来说这些林地面积太小，往 往不能吸引面积敏感型的鸟类 (Dawson et al, 1993 )。再加上污染、干扰等问题，使得城市中缺 少合适的筑巢地点, 而筑巢地点恰恰是影响鸟类营 巢集团的关键因素（Lim \& Sodhi, 2004）。因此随着 城市化程度的提高, 鸟类营巢集团的数量及各集团 的物种数都呈减少趋势, 并且在中度城市化区域也 未见其多样性明显增加, 这与本研究的预测一致, 且也在一定程度上反证了中度干扰理论（Connell, 1978)。

面对城市化的冲击，各营巢集团的反应并不一 致, 这与各集团对筑巢地资源的利用方式不同有 关。属于林冠枝干/灌草从营巢集团的鸟类既可以在 林冠枝干上筑巢, 也可以在灌草从中筑巢, 其对筑 巢地点的要求相对较低。因此在植被结构单一且干 扰严重的城区此集团鸟类的数量占优势。 
表 3 杭州地区乌类营巢集团在各栖息地中的平均比率 $(M \pm S E)$ 及其单因子方差分析的 $P$ 值

Tab. 3 Mean percentages $(M \pm S E)$ of the avian nesting guilds in different habitats in Hangzhou and the $p$ values of the one-way ANOVAs of mean percentages

\begin{tabular}{|c|c|c|c|c|c|c|c|c|}
\hline \multirow[b]{2}{*}{$\begin{array}{l}\text { 营巢集团 } \\
\text { Nesting guilds }\end{array}$} & \multicolumn{5}{|c|}{ 平均比率 Mean proportion (\%) } & \multirow[b]{2}{*}{$F$ 值 } & \multirow[b]{2}{*}{$\begin{array}{l}\text { 自由度 } \\
(d f)\end{array}$} & \multirow[b]{2}{*}{$P$ 值 } \\
\hline & $\begin{array}{c}\text { 城区 } \\
\text { Urban areas }\end{array}$ & $\begin{array}{c}\text { 城乡结合区 } \\
\text { Rural-urban } \\
\text { continuum areas }\end{array}$ & $\begin{array}{c}\text { 农田区 } \\
\text { Farmland areas }\end{array}$ & $\begin{array}{c}\text { 农林交错区 } \\
\text { Farmland and f } \\
\text { orestland mixed } \\
\text { areas }\end{array}$ & $\begin{array}{l}\text { 自然林区 } \\
\text { Forestland } \\
\quad \text { areas }\end{array}$ & & & \\
\hline $\mathrm{C}$ & $7.28 \pm 2.99$ & $10.74 \pm 2.95$ & $13.60 \pm 1.88$ & $15.16 \pm 2.33$ & $20.09 \pm 2.52$ & 3.503 & 4,85 & 0.011 \\
\hline S & $6.03 \pm 1.90$ & $9.96 \pm 2.33$ & $13.25 \pm 2.04$ & $35.98 \pm 3.60$ & $38.72 \pm 3.00$ & 33.623 & 4,85 & $<0.001$ \\
\hline $\mathrm{C} / \mathrm{S}$ & $17.52 \pm 2.35$ & $11.53 \pm 1.89$ & $12.14 \pm 2.32$ & $11.08 \pm 0.87$ & $10.25 \pm 1.81$ & 2.251 & 4,85 & 0.070 \\
\hline $\mathrm{NC}$ & $1.22 \pm 0.74$ & $0.14 \pm 0.14$ & $1.16 \pm 0.66$ & $1.38 \pm 0.53$ & $5.16 \pm 1.12$ & 7.388 & 4,85 & $<0.001$ \\
\hline B & $11.62 \pm 2.75$ & $10.36 \pm 2.06$ & $15.67 \pm 2.58$ & $9.21 \pm 1.52$ & $7.32 \pm 1.96$ & 1.996 & 4,85 & 0.102 \\
\hline $\mathrm{NC} / \mathrm{B}$ & $50.77 \pm 4.78$ & $50.21 \pm 4.94$ & $42.51 \pm 4.86$ & $24.50 \pm 3.12$ & $14.44 \pm 2.20$ & 15.494 & 4,85 & $<0.001$ \\
\hline $\mathrm{G}$ & - & $0.15 \pm 0.15$ & $1.31 \pm 0.52$ & $1.58 \pm 0.55$ & $3.00 \pm 0.42$ & 6.164 & 3,68 & 0.001 \\
\hline
\end{tabular}

(1)C：林冠枝干营巢集团（canopy nesters）：S：灌草丛营巢集团（shrubs nesters）；C/S：林冠枝干/灌草丛营巢集团（canopy/ shrub nesters）; NC: 自然洞穴营巢集团 (natural cavity nesters); B: 人工建筑营巢集团（buildings nesters); NC/B：自然洞穴/人工建筑营巢集团 (natural cavity/ buildings nesters); G：地面营巢集团（ground nesters); 一 表示该栖息地中无营巢集团分布（null guild in this habitat）。 (2)水面营巢集团与寄生集团因鸟类数量太少, 故未列入表中计算。

The data of water surface nesters and parasite nesters were not calculated here for the lack of the numbers of birds in the two guilds.

表 4 杭州地区乌类营巢集团在各栖息地中的平均比率与各栖息地城市化特征参数间的直线回归系数

Tab. 4 Regression coefficients for linear regression of mean percentages of the avian nesting guilds in Hangzhou versus urban characteristic parameters

\begin{tabular}{lccccc}
\hline $\begin{array}{c}\text { 营巢集团 } \\
\text { Nesting guilds }\end{array}$ & $\begin{array}{c}\text { 至中心城市市 } \\
\text { 中心距离 }(\mathrm{km}) \\
\text { Distance to city center }\end{array}$ & $\begin{array}{c}\text { 人为干扰 } \\
\text { Human disturbance }\end{array}$ & $\begin{array}{c}\text { 植被盖度 }(\%) \\
\text { Vegetation coverage }\end{array}$ & $\begin{array}{c}\text { 人工设施指数 }(\%) \\
\text { Building index }\end{array}$ & $\begin{array}{c}\text { 城市化综合指数 } \\
\text { Urbanization synthetic } \\
\text { index }\end{array}$ \\
\hline $\mathrm{C}$ & $0.487^{* *}$ & $-2.602^{* *}$ & $0.161^{* *}$ & $-0.133^{*}$ & $-0.410^{* *}$ \\
$\mathrm{~S}$ & $1.467^{*}$ & $-7.914^{*}$ & - & $-0.422^{*}$ & $-0.124^{*}$ \\
$\mathrm{C} / \mathrm{S}$ & - & - & $-0.089^{*}$ & - & - \\
$\mathrm{NC}$ & - & - & - & $-0.055^{*}$ & - \\
$\mathrm{B}$ & - & - & - & - & - \\
$\mathrm{NC} / \mathrm{B}$ & $-1.580^{*}$ & $8.468^{*}$ & $-472^{*}$ & $0.134^{* *}$ \\
\hline
\end{tabular}

(1) $P \leqslant 0.05 ;{ }^{* *} P \leqslant 0.01()$ 。

(2)C：林冠枝干营巢集团（canopy nesters）；S：灌草丛营巢集团（shrubs nesters）；C/S：林冠枝干/灌草丛营巢集团（canopy/ shrub nesters）; NC: 自然洞穴营巢集团 (natural cavity nesters); B: 人工建筑营巢集团 (buildings nesters); NC/B: 自然洞穴/人工建筑营巢集团 (natural cavity/ buildings nesters)。

(3)由于地面营巢集团、水面营巢集团及寄生集团在某些栖息地没有分布, 故未进行分析。

The data of ground nesters, water surface nesters and parasite nesters were not analyzed here for their lack in some habitats.

属于林冠枝干营巢集团的鸟类所占比例虽然 由于植被覆盖率下降而减少，但相对于灌木，城市 中有更多的乔木，使得林冠枝干营巢集团的鸟类有 更多筑巢地点可供选择, 并且因为筑巢地点较高, 林冠枝干营巢集团的鸟类也较不容易受到干扰 (Lim \& Sodhi, 2004)。

属于灌草丛营巢集团的鸟类筑巢依赖于灌草 丛, 但由于城市中的植被在垂直结构上往往缺乏灌 木层与草本层, 再加上在此层次人类活动频繁, 干 扰强烈, 使得灌草从营巢集团的鸟类很难找到合适 的筑巢地点。所以, 灌草丛营巢集团比林冠枝干营 巢集团对城市化更为敏感 (Park \& Lee, 2000)。

而对于属于地面营巢集团的鸟类来说, 城市化
造成的筑巢地资源缺失情况更为严重 (Luniak, 1981; Jokimäki, 1999)。Palomino \& Carrascal (2007) 的 研究发现, 尽管在轻度干扰的森林小路沿线和森林 野餐区的鸟类的多度和物种丰富度要高于森林深 处, 但地巢性鸟类的多度还是相对较低, 因为人类 的活动降低了地巢性鸟类的适宜度。

属于自然洞穴/人工建筑营巢集团的鸟类适应 强, 无论是树洞、泥洞、岩壁凹陷、或者人工建筑 的裂隙、孔洞中都能筑巢繁殖 (Kang et al, 1990), 城市中常见的麻雀、山麻雀便属于此集团。而且本 研究发现, 随着城市化程度的提高, 此集团鸟类的 筑巢地点也逐渐从自然洞穴过渡到了人工建筑。因 此在城市化进程中, 当其余集团逐渐退出时, 该集 
团便占据了主导地位。有研究证明洞巢鸟对城市化 的反应是最不敏感的 (Park \& Lee, 2000), 而 Chace \&Walsh（2006）也认为城市化倾向于选择洞巢鸟。

属于自然洞穴营巢集团的鸟类因为只能在自 然栖息地中的洞穴中筑巢, 故在城市化程度高的栖 息地中数量很少, 而其在自然林区的数量则要远大 于其它栖息地。特别是自己开丵树洞的鸟类集团, 往往在城市中缺失（Chace \& Walsh, 2006）。

属于人工建筑营巢集团的鸟类有家燕与金腰 燕, 它们倾向于选择农居的屋檐及房椽处作为营巢 地点。对于城市化程度更高的城区与城乡结合区来 说, 其干扰太大, 而对于更自然的农林交错区和自 然林区来说, 适合筑巢的建筑又太少, 因此, 此集 团所占比例在农田区达到峰值。

可见, 在本地区城市化对地面营巢集团和灌草 从营巢集团的影响最大, 对林冠枝干营巢集团也有 较大影响, 但不如前两者明显, 这也证实了本研究 先前的预测。而林冠枝干/灌草丛营巢集团则对城市 化比较适应。对于洞穴营巢集团, 情况则比较复杂, 其中只能利用自然洞穴的鸟类随着城市化程度提 高, 数量大减; 而既能利用自然洞穴, 又能利用人 工设施筑巢的鸟类则似乎得益于城市化, 随着城市 化的提高而逐渐占优。

\section{2 鸟类营巢集团分布的影响因素}

植被盖度是营巢集团分布的影响因素, 因为大 多数营巢集团的鸟类筑巢地点依赖于植被。 Sandström et al (2006) 的研究就证实树数量与洞巢 鸟数量有显著的相关性。另外 Jokimäki（1999）和

\section{参考文献:}

Allen AP, O’ Connor RJ. 2000. Hierarchical correlated of bird assemblage structure on northeastern U.S.A. lakes. Environ [J]. Monitor Assess, 62: 15-37.

Beissinger SR, Osborne DR. 1982. Effects of urbanization on avian community organization $[\mathrm{J}]$. The Condor, 84: 75-83

Blair RB. 1996. Land use and avian species diversity along an urban gradient [J]. Ecological Applications, 6(2): 506-519.

Blair RB. 1999. Birds and butterflies along an urban gradient [J]. Ecological Applications, 9(1): 164-170.

Blair R. 2004. The effect of urban sprawl on birds at multiple levels of biological $[\mathrm{J}]$. Ecology and Society, 9(5): 2 [online] URL: http://www.ecologyandsociety.org/vo19/iss5/art2

Bolger DT, Scott TA, Rotenberry JT. 1997. Breeding bird abundance in an urbanizing landscape in coastal southern California[J]. Conservation Biology, 11(2): 406-421.

Canterbury GE, Martin TE, Petit DR, Petit LJ, Bradford FB. 2000. Bird communities and habitat as ecological indicators of forest condition in regional monitoring [J]. Conservation Biology, 14(2): 544-558.
Villard et al（1999）则发现不论在林地栖息地还是 城市栖息地, 林地面积与鸟类群落的多样性都具有 正相关关系。不仅鸟类群落的丰富度与植被有关， 其密度也会随着植被的减少而减少（Mills et al, 1989; Rottenborn, 1999)。

建筑面积比例也是营巢集团分布的影响因数 之一, 因为许多适应城市化的鸟类往往会利用人工 设施筑巢。Blair (1996)、Chen et al (2000)及 Lim \& Sodhi (2004)的研究证实建筑面积比例对鸟类群落 的多样性及分布有显著影响。

人为干扰也会影响鸟类营巢集团的分布。比如 Wang et al（2008）的研究就发现人为干扰强度与喜 鹊巢高度有显著正相关关系。

总之, 城市化改变了自然栖息地的结构, 使得 鸟类营巢地点发生了变化。从而对鸟类营巢集团产 生了影响。城市中灌草层的缺失使得灌丛鸟和地巢 性鸟类减少甚至消失，较低的植被覆盖率也使得一 切依赖于植被筑巢的鸟类在城市中所占比重下降， 而城市化所带来的大量人工设施及建筑则为洞巢 鸟提供了大量合适的筑巢地点, 使此集团在城市中 占有优势地位。因此, 对于城市规划及建设来说, 应尽可能为鸟类保留或提供多样的筑巢地点, 具体 来说, 应尽可能保留本地的自然植被及其结构, 而 在进行城市绿化时, 也应尽量种植本地种, 并提高 灌木层和草本层的盖度。这样不仅能为依赖植被筑 巢的鸟类提供更多的筑巢地点, 也为其它他类提供 了食物资源及避难场所, 而这些能有效地提高城市 鸟类的丰富度及多样性。

Chace JF, Walsh JJ. 2006. Urban effects on native avifauna: A review [J]. Landscape and Planning, 74: 46-69.

Chen SH, Ding P, Zheng GM, Zhuge Y. 2000. Impacts of urbanization on the wetland water-bird communities in Hangzhou [J]. Zool Res, 21(4): 279-285. [陈水华, 丁 平, 郑光美, 诸葛阳. 2000. 城市化对杭州市 湿地水鸟群落的影响研究. 动物学研究, 21(4): 279-285.]

Chen FG, Luo SY. 1998. Fauna Sinica Aves Vol.9 [M]. Beijing: Science Press. [陈服官, 罗时有. 1998. 中国动物志 - 鸟纲: 第九卷, 雀形 目・太平鸟科一一岩翏科. 北京: 科学出版社.]

Cheng Tso-Hsin. 1978. Fauna Sinica Aves Vol.4 [M]. Beijing: Science Press. [郑作新. 1978. 中国动物志 - 鸟纲: 第四卷, 鸡形目. 北京: 科学出 版社.]

Cheng Tso-Hsin. 1979. Fauna Sinica Aves Vol.2 [M]. Beijing; Science Press. [郑作新. 1979. 中国动物志 - 鸟纲: 第二卷, 雁形目. 北京: 科学出 版社.]

Cheng Tso-Hsin, Long ZY, Zheng BF. 1987. Fauna Sinica Aves Vol.11 [M]. Beijing: Science Press. [郑作新, 龙泽虞, 郑宝㐘. 1987. 中国动物 志 - 鸟纲: 第十一卷, 雀形目 - 暡科: II 画眉亚科. 北京: 科学出版 
社.]

Cheng Tso-Hsin, Xian YH, Guan GX, 1991. Fauna Sinica Aves Vol.6 [M]. Beijing: Science Press. [郑作新, 冼耀华, 关贯勋. 1991. 中国动物 志 - 鸟纲: 第六卷, 鸽形目 - 鹦形目・鹃形目 - 鸮形目. 北京: 科学 出版社.]

Cheng Tso-Hsin, Long ZY, Lu TC. 1995. Fauna Sinica Aves Vol.10 [M]. Beijing; Science Press. [郑作新, 龙泽虞, 卢汰春. 1995. 中国动物志. 鸟纲: 第十卷, 雀形目 - 䴔科: I. 鸫亚科. 北京: 科学出版社.]

Cheng Tso-Hsin. 1997. Beijing: Science Press. [郑作新. 1997. 中国动物 志 - 鸟纲: 第一卷第一部, 中国鸟纲绪论, 第二部潜鸟目、鹈形目、 鹳形目等五目. 北京: 科学出版社.]

Clergeau P, Savard JPL, Mennechez G, Falardeau G. 1998. Bird abundance and diversity along an urabn-rural gradient: A comparative study between tow cities on different continents $[\mathrm{J}]$. The Condor, 100: 413-425.

Connell JH. 1978. Diversity in tropical rain forests and coral reefs [J]. Science, 199: 1302-1310.

Dawson DK, Darr LJ, Robbins CS. 1993. Predicting the distribution of breeding forest birds in a fragmented landscape [J]. Trans North $\mathrm{Am}$ Wildl Nat Resour Conf, 58: 35-43.

DeGraaf RM, Wentworth JM. 1986. Avian guild structure and habitat associations in suburban bird communities [J]. Urban Ecology, 9: 399-412.

Fernández-Juricic E. 2000. Avifaunal use of wooded streets in an urban landscape [J]. Conservation Biology, 14: 513-521.

Fontaine JJ, Martel M, Markland HM, Niklison AM, Decker KL, Martin TE. 2007. Testing ecological and behavioral correlates of nest predation [J]. Oikos, 116: 1887-1894.

Fu TS, Song YJ, Gao W. 1998. Fauna Sinica Aves Vol.14 [M]. Beijing: Science Press. [傅桐生, 宋榆钧, 高 纬. 1998. 中国动物志 - 鸟纲: 第十四卷, 雀形目 - 文鸟科, 雀科. 北京: 科学出版社.]

Goldstein EL, Gross M, DeGraaf RM. 1986. Breeding birds and vegetation: A quantitative assessment [J]. Urban Ecology, 9: 377-385.

Green DM, Backer MG. 2003. Urbanization impacts on habitat and bird communities in a Sonoran desert ecosystem [J]. Landscape and Urban Planning, 63: 225-239.

Jokimäki J, Suhonen J. 1998. Distribution and habitat selection of wintering birds in urban environments [J]. Landscape Urban Plann, 39: 253-263.

Jokimäki J. 1999. Occurrence of breeding bird species in urban park: Effects of park structure and broadscale variables [J]. Urban Ecosystems, 3: 21-34.

Kang N, Sigurdsson JB, Hails CJ, Counsilman JJ. 1990. Some implications of resource removal in the control of Mynas (Acridotheres spp.) in Singapore [J]. Malayan Nat J, 44: 103-108.

Li GY, Zheng BL, Liu GZ, 1982. Fauna Sinica Aves Vol.13 [M]. Beijing: Science Press. [李桂坦, 郑宝秉, 刘光佐. 1982. 中国动物志 - 鸟纲: 第十三卷, 雀形目 (山雀科——绣眼鸟科). 北京: 科学出版社.]

Li PF, Li W, Zhou W, Wang CF, Cai HY. 2004. Ecological habits of bird of hawk family in Baishilazi region [J]. Journal of Liaoning Forestry Science \& Technology, 3: 5-8. [李佩福, 李 伟, 周 威, 王成福, 蔡 洪岩. 2004. 白石砬子地区鹰科鸟类生态习性研究. 辽宁林业科技, 3: 5-8.]

Li XT. 2004. Raptors of China [M]. Beijing: China Forestry Publishing House. [李湘涛. 2004. 中国猛禽. 北京: 中国林业出版社.]

Lim HC, Sodhi NS. 2004. Responses of avian guilds to urbanization in a tropical city [J]. Landscape and Urban Planning. 66: 199-215.

Lindsay AR, Gillum SS, Meyer MW. 2002. Influence of lakeshore development on breeding bird communities in a mixed northern forest [J]. Biological Conservation, 107: 1-11.

Luniak M. 1981. The birds of the park habitats in Warsaw [J]. Acta Ornithol, 18: $335-370$.

MacGregor-Fors I. 2007. Relation between habitat attributes and bird richness in a western Mexico suburb [J]. Landscape and Urban Planning, 84: 92-98.

McKinney ML. 2002. Urbanization, biodiversity, and conservation [J]. BioScience, 52: 883-890.

Mills SG, Dunning JB. Bates JB. 1989. Effects of urbanization on breeding bird community structure in southwestern desert habitats [J]. The condor, 91(2): 416-428.

Melles S, Glenn S, Marti K. 2003. Urban bird diversity and landscape complexity: Species-environment associations along a multiscale habitat gradient [J]. Conservation Ecology, 7(1): 5. [online] URL: http://www.consecol.org/vol7/iss1/art5

Palomino D, Carrascal LM. 2006. Urban influence on birds at a regional scale: A case study with the avifauna of northern Madrid province [J]. Landscape and Urban Planning, 77: 276-290.

Palomino D, Carrascal LM. 2007. Impact of recreation on forest bird communities: Non-detrimental effects of trails and picnic areas [J]. Acta Zoologica Sinica, 53(1): 54-63.

Park CR, Lee WS. 2000. Relationship between species composition and area in breeding birds of urban woods in Seoul, Korea [J]. Landscape Urban Plann, 51: 29-36.

Rosenberg KV, Terrill SB, Rosengerg GH. 1987. Value of suburban habitats to desert riparian birds [J]. Wilson Bulletin, 99: 643-654.

Rottenborn SC. 1999. Predicting the impacts of urbanization on riparian bird communities [J]. Biological Conservation, 88: 289-299.

Sandström UG, Angelstam P, Mikusiński G. 2006. Ecological diversity of birds in relation to the structure of urban green space [J]. Landscape and Urban Planning, 77: 39-53.

Sun MY, Li KQ, Zhu JJ, Kao CC, Sun XJ Zhou ZS. 2002. Reproduction habits of three species of woodpeckers and their prey on insects [J]. Forest Pest and Disease, 21(2): 12-14. [孙明荣, 李克庆, 朱九军, 考 持联, 孙晓君, 周作山. 2002. 三种啄木鸟的繁殖习性及对昆虫的 取食研究. 中国森林病虫, 21(2): 12-14.]

Villard MA, Trzcinski KM, Merriam G. 1999. Fragmentation effects on forest birds: Relative influence of woodland cover and configuration on landscape occupancy [J]. Conservation Biology. 13: 774-783.

Wang YP, Chen SH, Ding P. 2004. Effects of urbanization on the winter bird foraging guilds [J]. Journal of Zhejiang University(Science Edition), 31(3): 330-336. [ 王彦平, 陈水华,丁 平. 2004. 城市化对冬季鸟类 取食集团的影响. 浙江大学学报 (理学版), 31(3): 330-337.]

Wang YP, Chen SH, Jing PP, Ding P. 2008. Black-billed Magpies (Pica pica) adjust nest characteristics to adapt to urbanization in Hangzhou, China [J]. Can J Zool, 86: 868-874.

Zheng BL. 1985. [郑宝㐘. 1985. 中国动物志 - 鸟纲: 第八卷, 雀形目(阔 嘴鸟科一一和平鸟科). 北京: 科学出版社.]

Zheng GM, 1995. Ornithology[M]. Beijing: Beijing Normal University Press. [郑光美. 1995. 鸟类学. 北京: 北京师范大学出版社.]

Zheng GM, Zhang CZ. 2002. Birds in China [M]. Beijing: China Forestry Publishing House. [郑光美, 张词祖. 2002. 中国野鸟. 2002. 北京: 中国林业出版社.]

Zhuge Y, Jiang SR. 1983. A survey of bird in Hangzhou [J]. Journal of Hangzhou University, 10: 50-64. [诸葛阳, 姜仕仁. 1983. 杭州鸟类 调查. 杭州大学学报, 10: 50-64.] 